\title{
Multi-center observational study on the adherence, quality of life, and adverse events in lung cancer patients treated with tyrosine kinase inhibitors
}

J Oncol Pharm Practice 202I, Vol. 27(5) II47-II58 (C) The Author(s) 2020 Article reuse guidelines: sagepub.com/journals-permissions DOI: | 0.1 | $77 /|078| 5522094638$ | journals.sagepub.com/home/opp @SAGE

\author{
Jelena Rosentreter' (D), Jürgen Alt ${ }^{2}$, Marius Fried ${ }^{2}$, \\ Geothy Chakupurakal $^{3}$, Jan Stratmann ${ }^{4} \mathbb{D}$ and Irene Krämer'
}

\begin{abstract}
Introduction: Cancer patients tend to prefer oral instead of parenteral chemotherapy. To date, there is little evidence on the medication adherence in cancer patients. We investigated medication adherence to tyrosine kinase inhibitors in patients suffering from non-small cell lung cancer.

Methods: Tyrosine kinase inhibitor adherence was measured electronically by MEMS $^{\circledR}$ (medication event monitoring system) over at least six months. Adherence rates were calculated in terms of Dosing Compliance, Timing Compliance, Taking Compliance, and Drug Holidays. Patients were dichotomized as adherent when Dosing Compliance and Timing Compliance were $\geq 80 \%$, Taking Compliance ranged between 90 and II $0 \%$, and $<$ I Drug Holiday was registered. Quality of life was assessed by two questionnaires (EORTC QLQ-C30 version 3.0, EORTC QLQ-LCI3) at three time points. Adverse drug events were reported via patient diaries.

Results: Out of 32 patients enrolled, data from 23 patients were evaluable. Median Dosing Compliance, Taking Compliance, and Timing Compliance adherence rates of tyrosine kinase inhibitor intake amounted to $100 \%$, $98 \%$, and $99 \%$, respectively; Drug Holidays were observed in three patients. Four patients were dichotomized as nonadherent. Three of them had a twice-daily tyrosine kinase inhibitor regimen. Median quality of life scores amounted to 67 ( $\max .100$ ) and remained unchanged over the study period. Fatigue and rash were the most frequently reported adverse drug events.

Conclusion: Medication adherence of non-small cell lung cancer patients treated with tyrosine kinase inhibitors was extraordinarily high and is likely to support the effectiveness of tyrosine kinase inhibitor treatment and a good quality of life over a long period of time. Adherence facilitating information and education is especially relevant for patients taking tyrosine kinase inhibitors in a twice-daily regimen.
\end{abstract}

\section{Keywords}

Lung cancer, tyrosine kinase inhibitor, medication adherence rate, electronic adherence measurement, quality of life

Date received: 12 May 2020; revised: 4 July 2020; accepted: 7 July 2020

\section{Introduction}

Lung cancer is the most common cancer entity worldwide. It is associated with a high incidence and prevalence and ranks first and second in mortality statistics for men and women, respectively. ${ }^{1}$ Over $80 \%$ of patients suffer from non-small cell lung cancer (NSCLC), ${ }^{2}$ which is mostly diagnosed in advanced stages. Antitumor therapy of NSCLC changed a lot during the last decade. Currently, antineoplastic

\footnotetext{
'Department of Pharmacy, University Medical Center, Johannes Gutenberg University Mainz, Mainz, Germany

${ }^{2}$ Department of Internal Medicine III (Hematology, Oncology, Pneumology), University Medical Center, Johannes Gutenberg University Mainz, Mainz, Germany

${ }^{3}$ Practice for Hematology and Oncology, Koblenz, Germany

${ }^{4}$ Department of Internal Medicine II (Hematology, Oncology), Hospital of the Goethe University Frankfurt, Frankfurt am Main, Germany
}

\section{Corresponding author:}

Jelena Rosentreter, Department of Pharmacy, University Medical Center, Johannes Gutenberg University Mainz, Langenbeckstraße I, 55 I 3 I Mainz, Germany.

Email: rosentreter.jelena@gmail.com 
agents (e.g. platin derivatives, paclitaxel, gemcitabine, and pemetrexed), vascular endothelial growth factor (VEGF) inhibitors (e.g. bevacizumab), check point inhibitors (e.g. pembrolizumab, nivolumab), and oral tyrosine kinase inhibitors (TKIs) are utilized as mono or combination therapy. In patients with advanced NSCLC, first- and second-line therapy regimens are hallmarked by TKIs. Methods of molecular genetics are used to identify driver mutations in the tumor cells, such as EGFR (epidermal growth factor receptor) mutations or ALK (anaplastic lymphoma kinase) and ROS1 (reactive oxygen species) translocations, in order to initiate targeted therapy with the appropriate oral TKI. Afatinib, erlotinib, gefitinib, and osimertinib target EGFR; nintedanib inhibits angiokinase receptors; and alectinib, ceritinib, and crizotinib address ALK. ${ }^{3}$ The TKI medication is to be taken once daily (QD) except for alectinib, crizotinib, and nintedanib, which must be taken twice daily (BID). Only crizotinib, gefitinib, and osimertinib can be taken without regard to meals.

Cancer patients tend to prefer oral chemotherapy, because of fewer visits to in-/outpatient clinics and medication use at home instead of invasive parenteral administration. ${ }^{4,5}$ However, this type of treatment is also associated with increased patient responsibility, as effectiveness depends on correct and reliable intake, also known as medication adherence. Medication adherence is defined as the "degree to which the person's behavior corresponds with the agreed recommendations from a health care provider". ${ }^{6}$ Conventionally, patients are considered adherent if they take correctly more than $80 \%$ of the prescribed doses. ${ }^{7}$ Non-adherence can occur consciously or unconsciously and manifest itself in different forms: Patients might take no medication at all, take the wrong dose, chose the wrong time point for intake or discontinue the medication intake for several days (Drug Holidays) or ultimately (non-persistence). Treatment-related factors like a more complex regimen (e.g. BID vs. QD administration) are believed to influence patients' ability and intention of participation, resulting in lower adherence rates. ${ }^{8}$ Medication adherence can also be influenced adversely when the time of medication intake depends on the time of meal intake. ${ }^{9}$ Lack of adherence can reduce the effectiveness of therapy including quality of life $(\mathrm{QoL})^{6}$ and increase healthcare costs. ${ }^{10}$

Although there are intensive discussions about advantages and disadvantages of oral, targeted antitumor therapy compared to parenteral chemotherapy, ${ }^{5}$ there is little evidence of adherence in cancer patients. Adherence rates to oral anticancer therapy ranging from $46 \%$ to $100 \%$ were reported in a systematic review focusing on endocrine antitumor therapy and oral leukemia medication. In most cases, questionnaires were used to measure adherence. Seven out of 63 studies deployed electronic adherence measurement (medication event monitoring systems [MEMS]) and found adherence rates amounting to minimum 78\% and maximum $100 \% .{ }^{11}$ Another review dealt with six studies reporting adherence rates with oral anticancer medication assessed by MEMS amounting to $74 \%-$ $100 \%{ }^{12}$ To our knowledge, there are six studies published regarding medication adherence in NSCLC patients treated with TKIs, assessing erlotinib ${ }^{13-17}$ or erlotinib and crizotinib. ${ }^{18}$ Electronic adherence measurement with MEMS was used in only one of these studies. ${ }^{16}$ The studies are heterogeneous in terms of the study design, methods used to determine adherence, and the threshold used to define adherence. Of note, medium to high adherence rates $(72 \%-97 \%)$ with erlotinib were reported. When choosing the adequate therapy for NSCLC patients, prolonged survival and symptom palliation are the main therapeutic goals. Additionally, (moderate) enhancement or at least maintenance of patients' QoL should play a vital role. Unfortunately, QoL is not consistently assessed in lung cancer trials and results are difficult to compare. ${ }^{19}$ QoL may be affected by the severity and frequency of adverse drug events (ADEs). Patientreported outcomes on ADEs could improve the patient-physician communication as well as ADE management. ${ }^{20}$ Better knowledge about the anticancer treatment is associated with higher medication adherence. ${ }^{21}$

The aim of our study was to investigate the medication adherence, QoL, wellbeing, and occurrence of ADEs in NSCLC patients, featuring the eight TKIs approved for NSCLC therapy in Germany over a long-term period of six to nine months. Data were obtained by continuous objective electronic measurement of medication adherence and questionnaires on QoL, patient-reported outcomes on wellbeing and ADE occurrence as well as patients' knowledge about their TKI therapy.

\section{Material and methods}

\section{Study design}

The study was designed as a prospective, noninterventional, non-randomized, multicenter observational study for patients suffering from NSCLC. The study was approved by the Ethics Committee (Institutional Review Board) of the Federal State of Rhineland-Palatinate (Germany; reference number 837.114.17 (10948)) and the Ethics Committee of the Medical Faculty of Goethe University Frankfurt (Germany; reference number 322/17). German- 
speaking NSCLC patients aged $\geq 18$ years who were treated in the outpatient clinics of University Medical Centers of Mainz and Frankfurt (Germany), respectively, and an oncologists' office in Koblenz (Germany) were eligible for the study. Further inclusion criteria comprised intake of one of the eight TKIs approved for NSCLC treatment, i.e. afatinib, alectinib, ceritinib, crizotinib, erlotinib, gefitinib, nintedanib, and osimertinib. Patients could be pretreated with TKIs or start TKI therapy at the time point of enrollment. Any dose of TKI as well as a switch between the TKIs defined above was acceptable. The study was performed according to The Declaration of Helsinki and Good Clinical Practice Guidelines. Written informed consent was obtained from all patients. Patients were recruited by the attending physicians on occasion of outpatient visits. Enrollment took place between June 2017 and September 2018. The observation period of each patient was designated to be minimum six months and maximum nine months. After enrollment, the attending physician documented the patient's sociodemographic data (e.g. sex, age, smoking status), medical data (e.g. Eastern Cooperative Oncology Group (ECOG) performance status, date of initial NSCLC diagnosis, histology, driver mutation), and details about the TKI medication, such as type of TKI and dose, in a pre-printed form. During the subsequent visits, modifications of the TKI regimen (e.g. dose reduction, treatment interruption or switch to a different TKI) and the current ECOG performance status were documented by the responsible physicians in pre-printed forms. All forms were sent to the pharmacist responsible for evaluation via pre-stamped envelopes.

\section{Medication adherence}

Electronic adherence measurement was conducted using MEMS $^{\circledR}$ bottles (Medication Event Monitoring Systems from AARDEX Group, Belgium). The device consists of a pill bottle and a cap containing the electronic processor which records date and time of each opening. Bottle openings were considered equal to medication intake. The attending physician informed the patients about the features of the $\mathrm{MEMS}^{\circledR}$. Medication use was continuously monitored over a period of minimum six and maximum nine months in each patient. Patients who did not complete a sixmonth observation period were to be replaced according to the study design.

MEMS $^{\circledR}$ bottles were filled for each patient with the amount of the TKI needed for one month, labeled, and packed together with written instructions for MEMS ${ }^{\circledR}$ bottle use. All patients were supplied with the same size of MEMS $^{\circledR}$ bottles $(250 \mathrm{~mL})$. MEMS ${ }^{\circledR}$ bottles were only used as secondary package for the TKI dosage forms. As needed, the TKI blister packages (primary packages) were cut to fit into the MEMS $^{\circledR}$ bottle, avoiding damage. If the monthly TKI demand did not fit into a single MEMS $^{\circledR}$ bottle (i.e. alectinib, ceritinib, crizotinib, and nintedanib), additional refill packages and instructions were provided together with the filled bottle. Patients receiving afatinib refilled their MEMS $^{\circledR}$ bottle weekly (to account for the fact that the original packaging contains four individual bags with seven tablets each). Following each subsequent outpatient appointment (about once per month), refill packages covering the TKI demand for one month were sent to the patients.

Patients were asked to document any exceptional or missed MEMS ${ }^{\circledR}$ bottle openings, specifying the date and reason in a pre-printed form which was provided initially together with the MEMS $^{\circledR}$ bottle. Patients returned this form together with their MEMS ${ }^{\circledR}$ bottle during an outpatient visit at the end of the study period.

Data were retrieved from the MEMS $^{\circledR}$ cap with MEMS $^{\circledR} \quad 6$ USB Reader. MEMS ${ }^{\circledR}$ Adherence Software Version 4.3 (both from AARDEX Group, Belgium) was used to calculate and analyze adherence rates.

Adherence data were checked for plausibility and amended, if necessary, by comparing it to patients' written documentation (missing events could for example be explained by the remark "removal the day before due to business trip"). Information documented by the physicians was included to avoid calculation errors (e.g. periods without MEMS $^{\circledR}$ bottle openings explained by medication discontinuation due to ADEs).

Adherence rates. Adherence rates were calculated by the retrieved MEMS $^{\circledR}$ data:

- Dosing Compliance (DC) is defined as the proportion (\%) of days with correct drug intake (correct number of openings per day) within the observation period. Patients were dichotomized as dosing adherent when the DC was $\geq 80 \%$.

- Taking Compliance (TC) is defined as proportion $(\%)$ of doses taken in relation to the prescribed doses. TC was calculated by the number of documented openings and the number of expected openings (frequency of intake per day multiplied by the number of observation days). Patients were dichotomized as taking adherent when the TC ranged between $90 \%$ and $110 \%$.

- Timing Compliance (TiC) describes the proportion $(\%)$ of days with intake in the correct time interval, here defined as $24 \pm 6 \mathrm{~h}$ for once-daily intake and 
$12 \pm 3 \mathrm{~h}$ for twice-daily intake. The $\mathrm{TiC}$ rate was calculated by the number of days with correct timing intervals and the number of observation days. Patients were dichotomized as timing adherent when the $\mathrm{TiC}$ was $\geq 80 \%$.

- Drug Holidays (DH) are defined as the number of events (n) without intake for $>48 \mathrm{~h}$. Patients were dichotomized as adherent when $<1$ DH was registered. ${ }^{22}$

\section{Questionnaires}

Quality of life. Patients were asked to complete the German version of the written, health-related QoL questionnaire for cancer patients EORTC QLQ-C30 version $3.0^{23}$ as well as the supplementary module EORTC QLQ-LC13 ${ }^{24}$ specific to lung cancer patients. The EORTC QLQ-C30 comprises 30 questions, including multi-item functional and symptom scales, a Global Health (QL2) scale, and single-item measures. EORTC QLQ-LC13 comprises 13 questions (one symptom scale and 12 single items). Answers are evaluated as score value ranging from 0 to 100 . A score of 100 represents best function/QoL or highest symptomatic burden. ${ }^{23,24}$ The pre-printed questionnaires were sent to the patients on three occasions during the observation period: about one week $\left(t_{1}\right)$, four months $\left(t_{2}\right)$, and seven or nine months ( $\mathrm{t}_{3}$; if still taking part) after enrollment. Patients returned the questionnaires by mail with prestamped envelopes.

Patient-reported outcomes ("patient diary"). Patients were asked to document their wellbeing and the occurrence of ADEs in a pre-printed diary. The specifically designed forms covered a period of 28 days. Maximum nine forms were used by a single patient. For each day, the form comprised a Likert-type rating scale of five differently graded smileys. Patients were asked to tick each day one smiley that expressed their wellbeing best. Using the same form, ADEs typical for TKI therapy, i.e. nausea/vomiting, rash, mucositis, diarrhea, and fatigue were documented. Patients were asked to mark, on daily basis, each ADE that occurred.

Patients' knowledge about TKI therapy. Two weeks after enrollment, patients were contacted for a telephone interview. It was based on a self-designed questionnaire regarding patients' knowledge on different aspects of TKI therapy, i.e. name and strength of the prescribed TKI, daily dosage and timing of intake in relation to meals. Responses were compared to correct answers, deducted from patients' medical history and the Summary of Product characteristics (SmPC). The numbers of correct and wrong answers were analyzed.

\section{Statistical analysis}

Statistical analysis was planned in cooperation with the Institute of Medical Biometry, Epidemiology and Informatics (IMBEI), University Medical Center, Mainz, Germany. A sample size of 50 patients was specified in consideration of the potential number of eligible patients, feasibility, and probability of valid results. The adherence rates (DC, TC, TiC, and $\mathrm{DH}$ ) and patients' sociodemographic and medical parameters were analyzed as median, minimum, and maximum rates. QoL questionnaires were analyzed according to the EORTC manual: The raw score (average of the items contributing to a certain scale) was standardized by using linear transformation, resulting in a final score ranging from 0 to $100 .{ }^{25}$ Median, minimum, and maximum QoL scores were calculated at the three time points. The difference (\%) between an individual patient's QL2 score at $t_{1}$ and $t_{3}$ was analyzed. A difference of $\geq \pm 10 \%$ in the QL2 score was estimated as clinically significant. ${ }^{26}$

The smileys documented in the patient diaries were graded from happiest ( 0 points) to saddest (4 points). The sum per patient over a 28-day period ("monthly score") could thus range from 0 points (best wellbeing) to a maximum of 112 points (worst wellbeing).

Each ADE marked by the patient in the diary was scored with one point. For each 28-day period, these points were summated (minimum 0 points, maximum 28 points per ADE). The number of patients with low ADE burden (defined as 0 points per ADE per month) and the number of patients with high ADE burden (defined as $\geq 15$ points per ADE per month, i.e. occurrence rate $>50 \%$ per month) were analyzed. All calculations were performed with MS Excel 2010.

\section{Results}

\section{Study population}

A total of 32 patients were enrolled in three centers. Seven patients did not complete the study over a minimum period of six months, most frequently due to progressive disease, resulting in discontinuation of TKI treatment. In addition, two patients did not return the $\mathrm{MEMS}^{\circledR}$ bottles at the end of the observation period. Thus, the data of 23 patients ( 13 female, 10 male) were analyzed per protocol. The median observation period per patient amounted to eight months (251 days). In total, 21 patients were taking part over a period longer than seven months and 14 patients longer than eight months. Sociodemographic and medical data characterizing the study population are shown in Table 1. Mutation of the EGFR was dominant and consequentially afatinib and osimertinib were most 
Table I. Sociodemographic and medical data of NSCLC patients who completed the study ( $\mathrm{n}=23$ patients).

\begin{tabular}{|c|c|}
\hline Age mean (min-max) (years) & $65(40-81)$ \\
\hline Sex female/male $(n)$ & $13 / 10$ \\
\hline (Former) smoker (n) & 13 \\
\hline Patients with ECOG performance status 0/I/2 (n) & $13 / 7 / 3$ \\
\hline Patients with stage IV cancer at initial diagnosis (n) & 20 \\
\hline Patients with histology of adenocarcinoma (n) & 22 \\
\hline \multicolumn{2}{|l|}{ Patients with driver mutation at initial diagnosis (n) } \\
\hline EGFR & 17 \\
\hline ALK & 2 \\
\hline ROS & 2 \\
\hline Other & 2 \\
\hline Period between initial diagnosis and enrollment, median (min-max) (months) & $21(0-107)$ \\
\hline \multicolumn{2}{|l|}{ Characteristics of TKI treatment } \\
\hline Patients pretreated with TKI before enrollment $(n)$ & 22 \\
\hline TKI treatment duration before enrollment, median (min-max) (months) & $13(0-83)$ \\
\hline Patients with one TKI switch before enrollment $(n)$ & 8 \\
\hline Patients with two TKI switches before enrollment (n) & I \\
\hline \multicolumn{2}{|l|}{ Patients treated with TKIs at enrollment $(n)$} \\
\hline Afatinib & II \\
\hline Osimertinib & 6 \\
\hline Crizotinib & 2 \\
\hline Alectinib, Ceritinib, Gefitinib, Nintedanib & I each \\
\hline Erlotinib & 0 \\
\hline Patients with reduced TKI dosage at enrollment $(n)$ & 13 \\
\hline Patients with TKI switch during observation time (n) & 5 \\
\hline
\end{tabular}

NSCLC: non-small cell lung cancer; EGFR: epidermal growth factor receptor; ALK: anaplastic lymphoma kinase; ROS: reactive oxygen species; ECOG: Eastern Cooperative Oncology Group; TKI: tyrosine kinase inhibitor.

often prescribed. The median duration of TKI treatment prior to enrollment amounted to 13 months. Of note, one patient received TKIs for almost seven years prior to study inclusion. Only one patient was enrolled on occasion of TKI treatment initiation. A TKI switch took place in nine patients prior to enrollment, while five patients had their TKI switched during the study period. A reduced TKI dosage at enrollment was observed in 13 patients.

\section{Medication adherence}

The adherence rates determined in NSCLC patients taking TKIs over a period of about eight months were extraordinarily high (median DC rate $100 \%$, TC rate $98 \%$, TiC rate $99 \%, \mathrm{DH}$ frequency 0 , see Table 2 ). Of note, the patient taking TKIs in the eighth year achieved a DC rate of $100 \%$. Adherence rates of patients taking the TKI twice daily were lower than those of patients taking the TKI once daily. TC, DC, and $\mathrm{TiC}$ rates of the individual patients are shown in Figures 1 and 2.

Four patients were dichotomized as nonadherent regarding one or multiple specifications of adherence rates. Two of them were dichotomized as non-adherent regarding all types of adherence rates.
Table 2. Adherence rates with TKI in NSCLC patients determined by MEMS $^{\circledR}(n=23$ patients).

\begin{tabular}{lll}
\hline Dosing Compliance rate & Median (\%) & 100 \\
& Min-Max (\%) & $75-100$ \\
Taking Compliance rate & Median (\%) & 98 \\
& Min-Max (\%) & $85-106$ \\
Timing Compliance rate & Median (\%) & 99 \\
& Min-Max (\%) & $48-100$ \\
Drug Holiday frequency & $\begin{array}{c}\text { Median (number } \\
\text { of events) }\end{array}$ & 0 \\
& $\begin{array}{c}\text { Min-Max (number } \\
\text { of events) }\end{array}$ & $0-6$ \\
\hline
\end{tabular}

TKI: tyrosine kinase inhibitor; NSCLC: non-small cell lung cancer; MEMS: medication event monitoring systems.

One patient was dichotomized as non-adherent regarding $\mathrm{TC}$ and $\mathrm{TiC}$ and one patient was dichotomized as non-adherent regarding DH only. All three patients dichotomized as non-adherent for $\mathrm{DC}, \mathrm{TC}$, or $\mathrm{TiC}$ belonged to the twice-daily dosing group. Two of them had to take more than one capsule per dose interval.

\section{Questionnaires}

Quality of life. The QoL questionnaires were returned by 21 and 22 out of 23 patients at $t_{1}$ and $t_{2}$ respectively, 


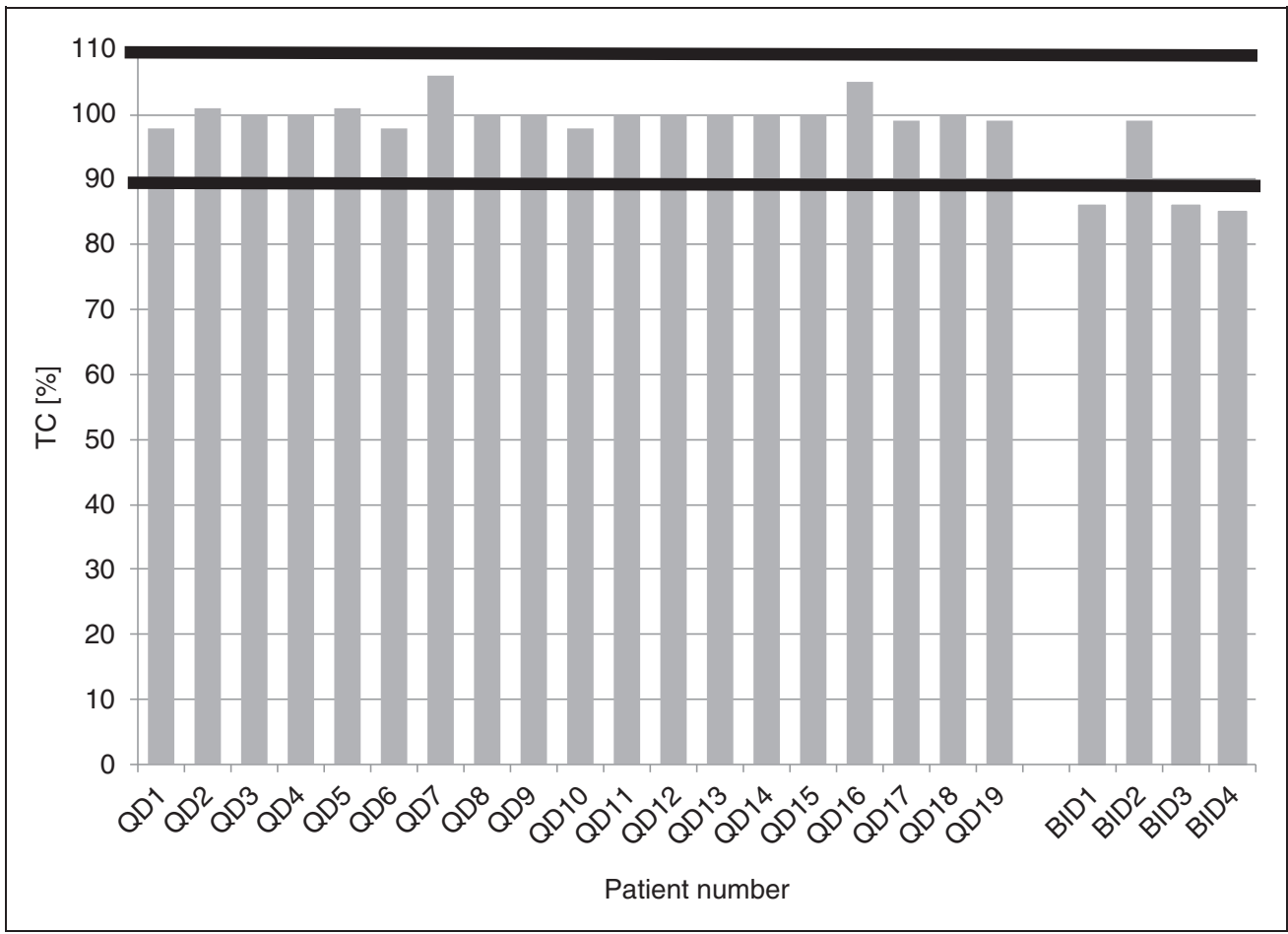

Figure I. Taking Compliance (TC) rates of patients enrolled, grouped by once-daily administration (QD) and twice-daily administration (BID); vertical lines $=$ threshold $\mathrm{TC} \geq 90 \%$ and $\leq \mathrm{II} 0 \%$.

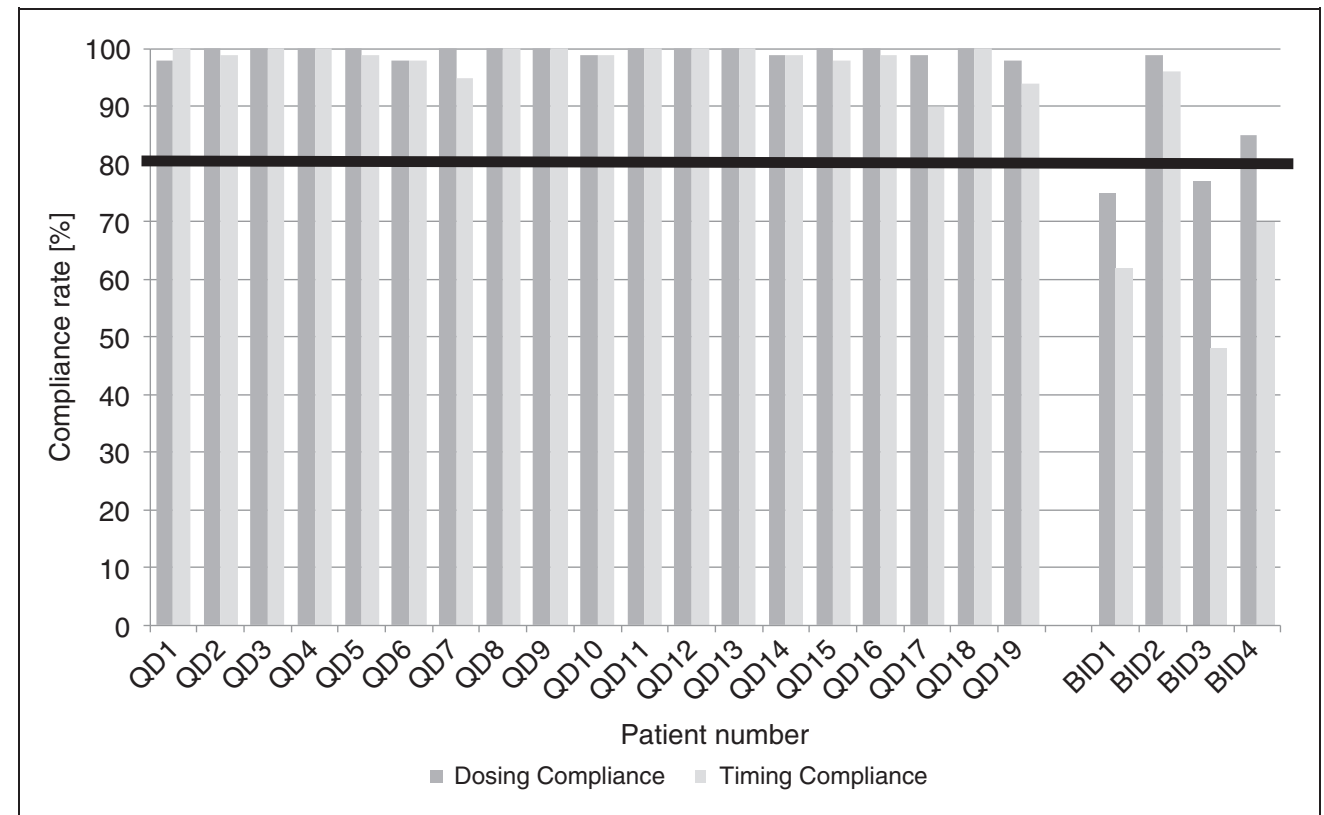

Figure 2. Dosing Compliance (DC) and Timing Compliance (TiC) rates of the individual patients enrolled, grouped by once-daily administration (QD) and twice-daily administration (BID); vertical line $=$ threshold $\mathrm{DC} / \mathrm{TiC} \geq 80 \%$.

and by 18 out of 21 patients at $t_{3}$ (for detailed results, see Table 3). On the level of the patient group, the median QL2 score amounted to 67 and remained unchanged at each time point. On the individual patient level, QL2 scores remained unchanged between $\mathrm{t}_{1}$ and $\mathrm{t}_{3}$ in four patients. A significant increase in QL2 scores from $t_{1}$ to $t_{3}$ was observed in eight patients, and a significant decrease in five out of 17 patients. Median 
Table 3. Quality of life determined by EORTC QLQ-C30 and EORTC QLQ-LCI 3 questionnaires at $t_{1}=$ study enrollment, $t_{2}=$ after 4 months, and $t_{3}=$ after 7-9 months.

\begin{tabular}{|c|c|c|c|c|}
\hline & & $t_{1}$ & $t_{2}$ & $t_{3}$ \\
\hline Number of NSCLC patients participating & & 21 & 22 & 18 \\
\hline \multicolumn{5}{|l|}{ EORTC QLQ-C30 } \\
\hline Global Health (QL2), median (min-max) score & & $67(33-92)$ & $67(42-100)$ & $67(33-100)$ \\
\hline \multirow[t]{5}{*}{ Functional scales, median (min-max) score } & Physical functioning (PF2) & $73(7-100)$ & $77(7-100)$ & $80(40-100)$ \\
\hline & Role functioning (RF2) & $67(0-100)$ & $67(0-100)$ & $58(0-100)$ \\
\hline & Emotional functioning (EF) & $79(42-100)$ & $75(8-100)$ & $67(50-100)$ \\
\hline & Cognitive functioning (CF) & $83(0-100)$ & $83(33-100)$ & $83(33-100)$ \\
\hline & Social functioning (SF) & $67(0-100)$ & $67(0-100)$ & $67(17-100)$ \\
\hline \multirow[t]{9}{*}{ Symptomatic scales, median (min-max) score } & Fatigue (FA) & $44(0-100)$ & $44(0-78)$ & $33(0-78)$ \\
\hline & Nausea/vomiting (NV) & $0(0-50)$ & $0(0-33)$ & $0(0-17)$ \\
\hline & Pain (PA) & $17(0-100)$ & $17(0-83)$ & $0(0-67)$ \\
\hline & Dyspnea (DY) & $33(0-100)$ & $33(0-67)$ & $33(0-67)$ \\
\hline & Insomnia (SL) & $33(0-100)$ & $33(0-67)$ & $33(0-67)$ \\
\hline & Appetite loss (AP) & $0(0-67)$ & $0(0-67)$ & $0(0-67)$ \\
\hline & Constipation (CO) & $0(0-100)$ & $0(0-67)$ & $0(0-100)$ \\
\hline & Diarrhea (DI) & $33(0-100)$ & $33(0-100)$ & $33(0-67)$ \\
\hline & Financial difficulties (FI) & $0(0-100)$ & $0(0-100)$ & $0(0-67)$ \\
\hline \multicolumn{5}{|l|}{ EORTC QLQ-LCI3 } \\
\hline \multirow[t]{10}{*}{ Symptomatic scales, median (min-max) score } & Dyspnea (LCDY) & $22(0-78)$ & $17(0-78)$ & II (0-67) \\
\hline & Coughing (LCCO) & $33(0-67)$ & $33(0-67)$ & $33(0-67)$ \\
\hline & Hemoptysis (LCHA) & $0(0-7)$ & $0(0-0)$ & $0(0-0)$ \\
\hline & Sore mouth (LSM) & $0(0-100)$ & $17(0-67)$ & $0(0-67)$ \\
\hline & Dysphagia (LCDS) & $0(0-100)$ & $0(0-33)$ & $0(0-67)$ \\
\hline & Peripheral neuropathy (LCPN) & $0(0-67)$ & $0(0-100)$ & $33(0-100)$ \\
\hline & Alopecia (LCHR) & $0(0-100)$ & $0(0-100)$ & $0(0-67)$ \\
\hline & Pain in chest (LCPC) & $0(0-100)$ & $0(0-100)$ & $0(0-33)$ \\
\hline & Pain in arm/shoulder (LCPA) & $0(0-33)$ & $0(0-100)$ & $33(0-100)$ \\
\hline & Pain in other parts (LCPO) & $0(0-67)$ & $33(0-100)$ & $33(0-67)$ \\
\hline
\end{tabular}

NSCLC: non-small cell lung cancer.

scores for the functional scales varied between 58 and 83 during the three time points, while the median scores of the symptomatic scales mostly remained unchanged. The scores per item were quite different, ranging from 0 (e.g. nausea/vomiting, appetite loss and financial difficulties) to the highest median score for fatigue (44) at $\mathrm{t}_{1}$ and $t_{2}$, respectively. According to the EORTC QLQLC13, coughing and "pain in other parts" were reported with the highest median score of 33 .

Patient-reported outcomes ("patient diary"). Out of 187 distributed reporting forms 175 were returned and analyzed. One patient did not return any of the nine forms provided. The monthly scores describing wellbeing on the individual patient's level ranged from 0 (optimum) to 66 .

All patients reported occurrence of ADEs. One patient reported only a single event of fatigue during the whole observation period. On the opposite, one patient reported three different ADEs (fatigue, mucositis, and rash) almost daily during the whole observation period. The least reported ADE overall is nausea/ vomiting, while fatigue and rash are the ADEs most often reported. For all types of ADEs and all months, there are more patients with low ADE burden than with high ADE burden. The number of patients with high ADE burden is highest for fatigue and rash. Table 4 details the frequency and longitudinal development of patient-reported ADE burden.

Patients' knowledge about TKI therapy. The telephone interview (duration: approximately 15 minutes) was conducted with 21 out of 23 patients. Five patients did not correctly name the TKI used, with three of them only making minor spelling mistakes. Four other patients did not know the correct dosage per tablet/ capsule. Another two patients did not correctly state the timing of TKI intake in relation to meals. Both of them were receiving afatinib at that point and were informed about correct intake during the interview. All patients knew how many doses they were supposed to take per day. 
Table 4. Occurrence of ADEs: Symptoms of any severity per month according to the patients' diaries.

\begin{tabular}{|c|c|c|c|c|c|c|c|c|c|c|c|}
\hline \multirow[b]{3}{*}{ Month } & \multirow{3}{*}{$\begin{array}{l}\text { Number of patient } \\
\text { diaries analyzed }(n)\end{array}$} & \multicolumn{10}{|c|}{ Number of patients reporting an ADE on days per month (n) } \\
\hline & & \multicolumn{2}{|c|}{ Nausea/Vomiting } & \multicolumn{2}{|l|}{ Rash } & \multicolumn{2}{|c|}{ Mucositis } & \multicolumn{2}{|c|}{ Diarrhea } & \multicolumn{2}{|l|}{ Fatigue } \\
\hline & & 0 days & $\geq 15$ days & 0 days & $\geq 15$ days & 0 days & $\geq 15$ days & 0 days & $\geq 15$ days & 0 days & $\geq 15$ days \\
\hline I & 22 & 19 & 0 & 12 & 4 & II & 5 & 7 & I & 9 & 7 \\
\hline 2 & 20 & 15 & 0 & II & 5 & 12 & 3 & 9 & 2 & 8 & 6 \\
\hline 3 & 21 & 16 & 0 & 12 & 5 & 12 & 3 & 8 & I & 9 & 6 \\
\hline 4 & 22 & 19 & 0 & 13 & 4 & 15 & 3 & II & I & 13 & 4 \\
\hline 5 & 22 & 21 & 0 & 12 & 8 & 14 & I & 11 & I & 13 & 3 \\
\hline 6 & 22 & 18 & 0 & 14 & 5 & 14 & 2 & 13 & 0 & 13 & 3 \\
\hline 7 & 19 & 15 & 0 & II & 5 & II & 2 & 9 & 0 & 12 & 4 \\
\hline 8 & 16 & 14 & 0 & 9 & 5 & 11 & 4 & 10 & 0 & 10 & 4 \\
\hline 9 & II & II & 0 & 6 & 3 & 9 & 0 & 8 & 0 & 7 & 2 \\
\hline
\end{tabular}

ADE: adverse drug event.

\section{Discussion}

The present study evaluated the medication adherence to TKIs in a small but well-characterized patient group for an extraordinary long median observation time of eight months. To our knowledge, this is the longest observation of medication adherence in TKI therapy reported. On the other hand, seven patients dropped out of the evaluation because of a shorter period of treatment. Compared to the study cohort analyzed by Timmers et al., ${ }^{16}$ our patients were of similar age but included more female patients, more (former) smokers, and a higher number of patients on reduced TKI dosage. The latter issue is attributable to the long median TKI treatment duration (13months) prior to enrollment. The high number of 14 patients having experienced at least one TKI switch either before or after enrollment is in accordance with the long period since initial NSCLC diagnosis and since TKI treatment initiation. It is noteworthy that in the present study not a single patient was treated with erlotinib, although in the current clinical practice guidelines none of the EGFR-targeting TKIs is favored. ${ }^{3}$ Previous studies on medication adherence in NSCLC patients were almost exclusively on erlotinib, ${ }^{13-18}$ only one of them also assessing three patients taking crizotinib. ${ }^{18}$ Today's diversification of targeted oral chemotherapy in NSCLC patients is reflected in the present study with eight TKIs and allows a better understanding of the current clinical practice.

\section{Medication adherence}

Adherence was assessed using MEMS $^{\circledR}$, which allows for detailed analysis of adherence patterns and is currently regarded as the gold standard of objective, electronic adherence measurement. ${ }^{27}$ Assessing adherence with MEMS $^{\circledR}$ is an indirect measurement method, i.e. without direct observation of patient's medication-taking behavior or analyzing blood samples. Time differences between TKI removal and actual ingestion or removal of less/more TKI than prescribed per dose are therefore possible scenarios. ${ }^{27}$ Conscious non-adherence (e.g. opening the bottle without removing medication or discarding the removed TKI instead of swallowing it) would most probably reduce the effectiveness of the anticancer therapy. ${ }^{28}$ This issue seems to be unlikely, as the patients' adherence patterns corresponded with the ECOG performance status documented by the physicians. Adjusting MEMS $^{\circledR}$ data according to patients' and physicians' written documentation on deviations of regular TKI intake (e.g. TKI removal from the MEMS $^{\circledR}$ bottle the day before, therapy interruption prescribed by the physician) allows for a more precise description of patients' adherence patterns. ${ }^{27}$ Unblinded adherence measurement by MEMS $^{\circledR}$ can influence adherence patterns for the better ("Hawthorne effect",29). This effect has been shown to wane after several weeks and an observation period of at least three months is in general sufficient to record an undistorted picture of the medication intake behavior. ${ }^{27,30}$

However, there are challenges inherent to $\mathrm{MEMS}^{\circledR}$ bottles. There is a risk of patients refraining to participate due to the device itself as transport is inconvenient and the bulky cap may be difficult to handle. High material costs and time-consuming service (preparation of monthly TKI refill packages) are to be considered. Systems like the "SmartBlister" (Confrérie Clinique, The Netherlands) could be a more convenient solution for future studies: The adherence measuring unit is contained in a thin, adhesive label, which is designed to fit the size of the medicinal product's blister. 
The observed high adherence rates are similar to those reported for oral anticancer therapy in gener$\mathrm{al}^{11,12}$ and for TKI therapy ${ }^{16}$ in particular. In agreement with other reports, ${ }^{31}$ older patients achieved higher adherence rates than the younger ones ( $\leq 45$ years). In contrast to other studies, ${ }^{11,16}$ we did not observe a decrease of the adherence rates over the prolonged observation period of eight months.

All patients dichotomized as non-adherent regarding DC, TC, and TiC were on a BID-TKI regimen and two of them had to take several capsules per dose interval. A third patient on a QD-TKI regimen and taking several capsules per dose interval showed $100 \%$ adherence rates and $0 \mathrm{DH}$. The finding that once-daily intake favors the medication adherence rates is in accordance with our previously published adherence data with immunosuppressive medication in liver transplant patients. ${ }^{32}$ In a previous review, ${ }^{31}$ a correlation between therapy-related factors (like intake regimen) and medication adherence did not get obvious. With respect to the limited number of patients observed, our findings nevertheless give a hint that patients with a twice-daily regimen might have an increased need for education and training on correct medication intake and medication adherence.

Out of the eight TKIs used, five require correct timing of intake in relation to meals and the filling stage of the stomach. Incorrect intake relative to the time-points of a meal can either diminish or elevate serum concentrations, leading to reduced efficacy or increased ADEs, thus possibly influencing outcome and QoL. Erlotinib is to be taken under fasting conditions and sub-optimal adherence was also significantly related with sub-optimal timing of the erlotinib intake. ${ }^{16}$ Based on the high adherence rates of our study population and the good knowledge about their TKI therapy, we assume that TKI intake was correctly timed. In patients with sub-optimal timing of erlotinib or afatinib intake related to the filling stage of the stomach, osimertinib might be a preferred option. Osimertinib is also approved for first-line therapy of EGFR-positive NSCLC and can be taken without regard to meals. ${ }^{3}$

\section{Questionnaires}

Quality of life. The health-related QoL questionnaires employed in the present study are the ones most frequently used in lung cancer trials. ${ }^{19}$ However, today the revised version EORTC QLQ-LC29 $9^{33}$ would be preferred. The results show that long-lasting TKI therapy allows patients to maintain their QoL over a long period despite the advanced stage of disease. The oral TKI intake was associated with higher QL2 scores than those reported by lung cancer patients undergoing i.v. chemotherapy ${ }^{34,35}$ and the observed QL2 scores come close to those of the normal population. ${ }^{35}$ Of note, we found a higher proportion of patients with consistently good or improved QoL than reported by Bezjak et al. for patients receiving erlotinib. ${ }^{36}$ It should be noted that those patients were TKI-naïve and treated with erlotinib without mandatory analysis of EGFR expression.

Patient-reported outcomes ("patient diary"). In the present study, fatigue and rash are the ADEs most often reported and most burdensome, which is in accordance with the findings in patients taking erlotinib. ${ }^{16}$ Patientreported outcomes are increasingly utilized to optimize the medical care of lung cancer patients. ${ }^{37}$ The discrepancy between the patient's and physician's perception about the occurrence of ADEs during erlotinib treatment ${ }^{16}$ underlines the importance of self-reporting by the patients. Daily documentation of wellbeing and ADEs by the patient provides a comprehensive picture of the disease course and can be used by the physician in the decision-making process regarding necessary dose adjustments or timely onset of ADE management. This can improve communication and satisfaction among the involved parties. ${ }^{37}$ However, daily documentation is associated with higher effort for both patients and health care providers. In the future, other self-reporting tools like the PRO-CTCAE questionnaire (patient-reported outcomes version of common terminology criteria for adverse events) ${ }^{38}$ or electronic applications should be tested.

Patients' knowledge about TKI therapy. To increase the benefit of oral anticancer therapy, patients need to be educated on the relevance of medication adherence, intake modalities, and self-management of ADEs. There is evidence that adequate information is associated with better medication adherence and QoL. ${ }^{38}$ Moreover, structured patient education can help to reduce the ADEs in patients receiving oral antineoplastic agents. ${ }^{39}$ The good knowledge about TKI therapy in our study population might be attributable to the longterm treatment with TKIs and the availability of specific patient information leaflets. In the future, health care professionals should focus on patients' knowledge about self-management of ADEs. To measure satisfaction with the information received, the Satisfaction with Information about Medicines Scale (SIMS questionnaire $)^{40}$ or the EORTC QLQ-INFO2 $5^{41}$ questionnaire can be used.

\section{Limitations}

The present study has certain limitations. The main issue is the limited number of participating patients. 
The envisaged goal of enrolling 50 patients was not met and related to the study design. The study was planned to last two years; thus, patient enrollment was terminated after 16 months. The number of patients fulfilling the inclusion criteria in the participating centers was lower than originally assumed. Due to inconsistent documentation, it is not possible to evaluate whether all eligible patients were invited to participate, how many of the invited patients refused to participate, and what the reasons for their refusal were. Since we included pretreated as well as TKI-naïve patients, the study cohort was heterogeneous in terms of TKI treatment duration prior to enrollment as well as time between patients' initial NSCLC diagnosis and enrollment. Conducting a study with only TKI-naïve patients would require multiple trial centers and a much longer recruiting period.

Participation was linked to additional efforts for the patients, especially the use of MEMS ${ }^{\circledR}$ bottle, the ongoing documentation of ADEs in a diary, and answering questionnaires. It can be further assumed that some of the invited patients refrained from participating due to the advanced stage of cancer and resulting life circumstances. A selection bias cannot be excluded. Attending physicians' motivation for study enrollment may also play a role: The surmount workload was not reimbursed. Additional obstacles were the high percentage rate of drop-outs caused by patients not returning their MEMS $^{\circledR}$ bottle and patients not completing the minimum observation period of six months, most frequently due to treatment discontinuation. In the study of Timmers et al., a total of 62 patients were included, of whom only 15 were on erlotinib treatment after four months. ${ }^{16}$ Therefore, a minimum observation period of three months would be more appropriate in future studies.

The objective method of adherence measurement was not completed with a subjective method by means of self-reporting, like the Morisky Medication Adherence Scale (MMAS) ${ }^{42}$ On one hand, that makes it difficult to compare our results. On the other hand, such self-reports depict adherence only at certain points in time. A gold standard of adherence measurement has not yet been determined for oral anticancer therapies. ${ }^{43}$

\section{Conclusion/outlook}

Medication adherence is a prerequisite to achieve clinical benefit with orally administered anticancer agents. NSCLC patients treated with targeted TKI therapy are able to accomplish high adherence rates, which is likely to support effectiveness and good QoL over a treatment period of more than six months. Adherence facilitating information and education is especially relevant for patients taking TKIs in a twice-daily regimen. Regarding ADE management, special attention should be paid to fatigue and rash. Further real-life studies should evaluate a bigger number of patients, include new TKIs approved for NSCLC treatment (like brigatinib, dacomitinib, lorlatinib), investigate the intake of TKIs in relation to meal intake, and combine electronic adherence measurement with patientreported adherence over periods of at least three months.

\section{Declaration of Conflicting Interests}

The author(s) declared no potential conflicts of interest with respect to the research, authorship, and/or publication of this article.

\section{Funding}

The author(s) received no financial support for the research, authorship, and/or publication of this article.

\section{ORCID iDs}

Jelena Rosentreter (D) https://orcid.org/0000-0001-7802-9135 Jan Stratmann (D) https://orcid.org/0000-0001-7726-6622 Irene Krämer (D) https://orcid.org/0000-0002-7576-3665

\section{References}

1. Bray F, Ferlay J, Soerjomataram I, et al. Global cancer statistics 2018: GLOBOCAN estimates of incidence and mortality worldwide for 36 cancers in 185 countries. $C A$ Cancer J Clin 2018; 68: 394-424.

2. American Cancer Society. Cancer facts \& figures 2019. Atlanta: American Cancer Society, 2019.

3. Planchard D, Popat S, Kerr K, et al. Metastatic nonsmall cell lung cancer: ESMO Clinical Practice Guidelines for diagnosis, treatment and follow-up. Ann Oncol 2018; 29: iv192-iv237.

4. Liu G, Franssen E, Fitch MI, et al. Patient preferences for oral versus intravenous palliative chemotherapy. J Clin Oncol 1997; 15: 110-115.

5. Eek D, Krohe M, Mazar I, et al. Patient-reported preferences for oral versus intravenous administration for the treatment of cancer: a review of the literature. Patient Prefer Adherence 2016; 10: 1609-1621.

6. World Health Organization. Adherence to long term therapies: evidence for action. Geneva: WHO, 2003.

7. Morrison A, Stauffer ME and Kaufman AS. Defining medication adherence in individual patients. Patient Prefer Adherence 2015; 9: 893-897.

8. Ruddy K, Mayer E and Partridge A. Patient adherence and persistence with oral anticancer treatment. $C A$ Cancer J Clin 2009; 59: 56-66.

9. Regnier Denois V, Poirson J, Nourissat A, et al. Adherence with oral chemotherapy: results from a qualitative study of the behaviour and representations of patients and oncologists. Eur J Cancer Care (Engl) 2011; 20: 520-527. 
10. Cutler RL, Fernandez-Llimos F, Frommer M, et al. Economic impact of medication non-adherence by disease groups: a systematic review. BMJ Open 2018; 8: e016982.

11. Greer JA, Amoyal N, Nisotel L, et al. A systematic review of adherence to oral antineoplastic therapies. Oncologist 2016; 21: 354-376.

12. Barillet M, Prevost V, Joly F, et al. Oral antineoplastic agents: how do we care about adherence? $\mathrm{Br} \mathrm{J}$ Clin Pharmacol 2015; 80: 1289-1302.

13. Boucher J, Lucca J, Hooper C, et al. A structured nursing intervention to address oral chemotherapy adherence in patients with non-small cell lung cancer. Oncol Nurs Forum 2015; 42: 383-389.

14. Gebbia V, Bellavia M, Banna GL, et al. Treatment monitoring program for implementation of adherence to second-line erlotinib for advanced non-small-cell lung cancer. Clin Lung Cancer 2013; 14: 390-398.

15. Engel-Nitz Nicole M, Nm Satram-Hoang S, Cao F, et al. Lung cancer: copayments and behavior following erlotinib formulary tier change. Am J Pharm Benef 2012; 4: SP6-SP16.

16. Timmers L, Boons C, Moes-Ten Hove J, et al. Adherence, exposure and patients' experiences with the use of erlotinib in non-small cell lung cancer. $J$ Cancer Res Clin Oncol 2015; 141: 1481-1491.

17. Hess LM, Louder A, Winfree K, et al. Factors associated with adherence to and treatment duration of erlotinib among patients with non-small cell lung cancer. JMCP 2017; 23: 643-652.

18. Torrecillas S, Perrot E, Gérinière L, et al. Croyances des patients envers les thérapies ciblées orales et leur influence sur l'observance dans le cancer broncho-pulmonaire une étude pilote prospective. Rev Pneumol Clin 2016; 72: 25-34.

19. Damm K, Roeske N and Jacob C. Health-related quality of life questionnaires in lung cancer trials: a systematic literature review. Health Econ Rev 2013; 3: 15.

20. Basch E, Iasonos A, McDonough T, et al. Patient versus clinician symptom reporting using the National Cancer Institute common terminology criteria for adverse events: results of a questionnaire-based study. Lancet Oncol 2006; 7: 903-909.

21. Verbrugghe $\mathbf{M}$, Verhaeghe $\mathrm{S}$, Lauwaert $\mathrm{K}$, et al. Determinants and associated factors influencing medication adherence and persistence to oral anticancer drugs: a systematic review. Cancer Treat Rev 2013; 39: 610-621.

22. Vrijens B and Goetghebeur E. Comparing compliance patterns between randomized treatments. Control Clin Trials 1997; 18: 187-203.

23. Aaronson NK, Ahmedzai S, Bergman B, et al. The European Organization for Research and Treatment of Cancer QLQ-C30: a quality-of-life instrument for use in international clinical trials in oncology. $J$ Natl Cancer Inst 1993; 85: 365-376.

24. Bergman B, Aaronson NK, Ahmedzai S, et al. The EORTC QLQ-LC13: a modular supplement to the EORTC core quality of life questionnaire (QLQ-C30) for use in lung cancer clinical trials. EORTC study group on quality of life. Eur J Cancer 1994; 30: 635-642.

25. Fayers PM, Aaronson NK, Bjordal K, et al. The EORTC QLQ-C30 scoring manual. 3rd ed. Brussels: European Organisation for Research and Treatment of Cancer, 2001.

26. Osoba D, Rodrigues G, Myles J, et al. Interpreting the significance of changes in health-related quality-of-life scores. J Clin Oncol 1998; 16: 139-144.

27. Denhaerynck K, Schäfer-Keller P, Young J, et al. Examining assumptions regarding valid electronic monitoring of medication therapy: development of a validation framework and its application on a European sample of kidney transplant patients. BMC Med Res Methodol 2008; 8: 5.

28. Timmers L, Boons C, Kropff F, et al. Adherence and patients' experiences with the use of oral anticancer agents. Acta Oncol 2014; 53: 259-267.

29. Partridge AH, Avorn J, Wang PS, et al. Adherence to therapy with oral antineoplastic agents. J Natl Cancer Inst 2002; 94: 652-661.

30. Deschamps AE, van Wijngaerden E, Denhaerynck K, et al. Use of electronic monitoring induces a 40-day intervention effect in HIV patients. $J$ Acquir Immune Defic Syndr 2006; 43: 247-248.

31. Gast A and Mathes T. Medication adherence influencing factors - an (updated) overview of systematic reviews. Syst Rev 2019; 8: 112.

32. Eberlin M, Otto $G$ and Krämer I. Increased medication compliance of liver transplant patients switched from a twice-daily to a once-daily tacrolimusbased immunosuppressive regimen. Transplant Proc 2013; 45: 2314-2320.

33. Koller M, Hjermstad MJ, Tomaszewski KA, et al. An international study to revise the EORTC questionnaire for assessing quality of life in lung cancer patients. Ann Oncol 2017; 28: 2874-2881.

34. Hung H-Y, Wu L-M and Chen K-P. Determinants of quality of life in lung cancer patients. J Nurs Scholarsh 2018; 50: 257-264.

35. Larsson M, Ljung L and Johansson BBK. Health-related quality of life in advanced non-small cell lung cancer: correlates and comparisons to normative data. Eur $J$ Cancer Care (Engl) 2012; 21: 642-649.

36. Bezjak A, Tu D, Seymour L, et al. Symptom improvement in lung cancer patients treated with erlotinib: quality of life analysis of the National Cancer Institute of Canada clinical trials group study BR.21. JCO 2006; 24: 3831-3837.

37. Bouazza YB, Chiairi I, El Kharbouchi O, et al. Patientreported outcome measures (PROMs) in the management of lung cancer: a systematic review. Lung Cancer 2017; 113: 140-151.

38. Boons C, Timmers L, van Schoor NM, et al. Patient satisfaction with information on oral anticancer agent use. Cancer Med 2018; 7: 219-228.

39. Riese C, Weiß B, Borges U, et al. Effectiveness of a standardized patient education program on therapy-related 
side effects and unplanned therapy interruptions in oral cancer therapy: a cluster-randomized controlled trial. Support Care Cancer 2017; 25: 3475-3483.

40. Horne R, Hankins M and Jenkins R. The Satisfaction with Information about Medicines Scale (SIMS): a new measurement tool for audit and research. Qual Health Care 2001; 10: 135-140.

41. Arraras JI, Greimel E, Sezer O, et al. An international validation study of the EORTC QLQ-INFO25 questionnaire: an instrument to assess the information given to cancer patients. Eur J Cancer 2010; 46: 2726-2738.
42. Morisky DE, Green LW and Levine DM. Concurrent and predictive validity of a self-reported measure of medication adherence. Med Care 1986; 24: 67-74.

43. Mislang AR, Wildes TM, Kanesvaran R, et al. Adherence to oral cancer therapy in older adults: the International Society of Geriatric Oncology (SIOG) taskforce recommendations. Cancer Treat Rev 2017; 575: $8-66$. 